\title{
Path Integral Quantization and Riemannian-Symplectic Manifolds
}

\author{
Sergei V. SHABANOV a, and John R. KLAUDER ${ }^{b}$ \\ ${ }^{a}$ Institute for Theoretical Physics, FU-Berlin, Arnimallee 14, Berlin, \\ D-14195, Germany \\ ${ }^{b}$ Departments of Physics and Mathematics, University of Florida, Gainesville, \\ FL-32611, USA
}

\begin{abstract}
We develop a mathematically well-defined path integral formalism for general symplectic manifolds. We argue that in order to make a path integral quantization covariant under general coordinate transformations on the phase space and involve a genuine functional measure that is both finite and countably additive, the phase space manifold should be equipped with a Riemannian structure (metric). A suitable method to calculate the metric is also proposed.
\end{abstract}

1. Most quantization formulations lead to correct results only when the system in question is expressed in one of a family of special (Cartesian) coordinate systems [1]. However, for Hamiltonian systems on a generic symplectic manifold, the canonical variables do not admit such special coordinates. Moreover classical Hamiltonian dynamics exhibits covariance under general coordinate transformations on the symplectic manifold, and, in this sense, is coordinate-free. This covariance is lost upon a formal phase-space path integral quantization. The reason is that the Hamiltonian action involves terms linear in the time derivatives and, therefore, its exponential (even in Euclidean time) does not induce a proper (normalizable, $\sigma$-additive) measure on the path space which is covariant under general coordinate transformations. Since the discovery of the Hamiltonian path integral by Feynman, the indisputable fact of its coordinate dependence has generally complicated any straightforward use of Hamiltonian path integrals in applications. Indeed, to justify any formal manipulation regarding the path integral measure, the operator formalism has generally been invoked. The aim of this letter is to resolve this long-standing problem and to establish a rigorous, coordinate-free path integral formalism for general Hamiltonian systems.

In [2] it was shown that the formal phase-space path integral measure can be made covariant under general coordinate transformations by means of a special regularization that involves an auxiliary Brownian motion on a flat phase space. The desired quantum mechanics is restored by taking the diffusion constant to infinity. The modified Hamiltonian path integral determines a stochastic process on the phase space in which the original first-order Lagrangian plays the role of the external potential. The stochastic process on a flat phase space remains flat under coordinate transformations. Thus one may change the variables in the regularized path integral according to the rules of the stochastic integral

\footnotetext{
${ }^{1}$ On leave from Laboratory of Theoretical Physics, JINR, Dubna, Russia.
} 
calculus, and at the end of calculations one has to take the infinite diffusion constant limit.

To be specific, and also to introduce notation, consider quantum mechanics in a flat phase space. Let $\hat{q}^{j}$ be a set of Cartesian coordinate canonical operators satisfying the Heisenberg commutation relations $\left[\hat{q}^{j}, \hat{q}^{k}\right]=i \stackrel{\circ}{\omega}^{j k}$. Here $\stackrel{\circ}{\omega}^{j k}=-\stackrel{\circ}{\omega}^{k j}$ is the canonical symplectic structure. We introduce the canonical coherent states as $|q\rangle \equiv e^{i q^{j} \stackrel{o}{\omega k} \hat{q}^{k}}|0\rangle$, where $\stackrel{\circ}{\omega}_{j n} \stackrel{\circ}{\omega}^{n k}=\delta_{j}^{k}$, and $|0\rangle$ is the ground state of a harmonic oscillator with unit angular frequency. Any state $|\psi\rangle$ is given as a function on phase space in this representation by $\langle q \mid \psi\rangle=\psi(q)$. A general operator $\hat{A}$ can be represented in the form $\hat{A}=\int d q a(q)|q\rangle\langle q|$, where $a(q)$ is the lower symbol of the operator and $d q$ is a properly normalized form of the Liouville measure. The function $A\left(q, q^{\prime}\right)=\left\langle q|\hat{A}| q^{\prime}\right\rangle$ is the kernel of the operator.

The main object of the path integral formalism is the integral kernel of the evolution operator

$$
K_{t}\left(q, q^{\prime}\right)=\left\langle q\left|e^{-i t \hat{H}}\right| q^{\prime}\right\rangle=\int_{q(0)=q^{\prime}}^{q(t)=q} \mathcal{D} q e^{i \int_{0}^{t} d \tau\left(\frac{1}{2} q^{j} \stackrel{\circ}{\omega}_{j k} \dot{q}^{k}-h\right)}
$$

Here $\hat{H}$ is the Hamiltonian, and $h(q)$ its symbol. The measure formally implies a sum over all phase-space paths pinned at the initial and final points, and a Wiener measure regularization implies the following replacement

$$
\mathcal{D} q \rightarrow \mathcal{D} \mu_{\nu}(q)=\mathcal{D} q e^{-\frac{1}{2 \nu} \int_{0}^{t} d \tau \dot{q}^{2}}=N_{\nu}(t) d \mu_{W}^{\nu}(q) .
$$

The factor $N_{\nu}(t)$ equals $2 \pi e^{\nu t / 2}$ for every degree of freedom, $d \mu_{W}^{\nu}(q)$ stands for the Wiener measure, and $\nu$ denotes the diffusion constant. We denote by $K_{t}^{\nu}\left(q, q^{\prime}\right)$ the integral kernel of the evolution operator for a finite $\nu$. The Wiener measure determines a stochastic process on the flat phase space. The integral of the symplectic one-form $\int q \stackrel{\circ}{\omega} d q$ is a stochastic integral that is interpreted in the Stratonovich sense. Under general coordinate transformations $q=q(\bar{q})$, the Wiener measure describes the same stochastic process on flat space in the curvilinear coordinates $d q^{2}=d \sigma(\bar{q})^{2}$, so that the value of the integral is not changed apart from a possible phase term. After the calculation of the integral, the evolution operator kernel is obtained by taking the limit $\nu \rightarrow \infty$. The existence of this limit, and also the covariance under general phase-space coordinate transformations, can be most easily proved through the operator formalism for the regularized kernel $K_{t}^{\nu}\left(q, q^{\prime}\right)$.

Note that the integral (11) with the Wiener measure inserted can be regarded as an ordinary Lagrangian path integral with a complex action, where the configuration space is the original phase space and the Hamiltonian $h(q)$ serves as a potential. Making use of this observation it is not hard to derive the corresponding Schrödinger-like equation

$$
\partial_{t} K_{t}^{\nu}\left(q, q^{\prime}\right)=\left[\frac{\nu}{2}\left(\partial_{q^{j}}+\frac{i}{2} \stackrel{\circ}{\omega}_{j k} q^{k}\right)^{2}-i h(q)\right] K_{t}^{\nu}\left(q, q^{\prime}\right),
$$

subject to the initial condition $K_{t=0}^{\nu}\left(q, q^{\prime}\right)=\delta\left(q-q^{\prime}\right), 0<\nu<\infty$. One can easily show that $\hat{K}_{t}^{\nu} \rightarrow \hat{K}_{t}$ as $\nu \rightarrow \infty$ for all $t>0$. The covariance under general coordinate 
transformations follows from the covariance of the "kinetic" energy of the Schrödinger operator in (3): The Laplace operator is replaced by the Laplace-Beltrami operator in the new curvilinear coordinates $q=q(\bar{q})$, so the solution is not changed, but written in the new coordinates. This is similar to the covariance of the ordinary Schrödinger equation and the corresponding Lagrangian path integral relative to general coordinate transformations on the configuration space: The kinetic energy operator (the Laplace operator) in the ordinary Schrödinger equation provides a term quadratic in time derivatives in the path integral measure which is sufficient for the general coordinate covariance [3]. We remark that the regularization procedure based on the modified Schrödinger equation (3) applies to far more general Hamiltonians than those quadratic in canonical momenta and leading to the conventional Lagrangian path integral.

The regularization procedure developed above deserves a further mathematical elaboration. To this end we point out that the operator $\hat{K}_{t}^{\nu}$ acts in a Hilbert space, denoted here and belows as $\mathcal{H}_{\nu}$, that is larger than the original Hilbert space $\mathcal{H}_{\infty}$. Indeed, consider canonical pairs $\left[\hat{Q}^{k}, \hat{P}_{n}\right]=i \delta_{n}^{k},\left[\hat{P}_{k}, \hat{P}_{n}\right]=\left[\hat{Q}^{k}, \hat{Q}^{n}\right]=0$ and sharp-eigenvalue states $\mid q)$ (observe the difference in notation!) being eigenstates of the complete set of commutative operators: $\left.\left.\hat{Q}^{k} \mid q\right)=q^{k} \mid q\right)$. These states form a basis in $\mathcal{H}_{\nu}$. So a resolution of unity reads $\left.\hat{\mathbb{1}}_{\nu}=\int d q \mid q\right)\left(q \mid\right.$ where $\left(q \mid q^{\prime}\right)=\delta\left(q-q^{\prime}\right)$. In constrast to $\left.\mid q\right)$, the coherent states $|q\rangle$ form a basis in the smaller space $\mathcal{H}_{\infty}$, though they carry the same label $q$. Note that the coherent states in $\mathcal{H}_{\nu}$ would have twice as many labels, say $p$ and $q$, associated with the canonical operators $\hat{P}$ and $\hat{Q}$, respectively. In $\mathcal{H}_{\nu}$ the original canonical operators can be represented as $\hat{q}^{k}=-\stackrel{\circ}{\omega}^{k n} \hat{P}_{n}+\frac{1}{2} \hat{Q}^{k}$, that is, we have a reducible representation of the original Heisenberg algebra. It is also important to observe that the extension of any opearator in $\mathcal{H}_{\infty}$ to the larger space $\mathcal{H}_{\nu}$ is by no means unique. This issue is not, however, our main concern.

To achieve a continuous-time regularization of the path integral measure, and thereby to make the latter mathematically well defined, it is sufficient to consider a specific extension of the unitary operator $\hat{K}_{t}=\exp (-i t \hat{H})$ which is induced by the extension of the Hamiltonian (cf. (3))

$$
\hat{H} \rightarrow \hat{H}_{\nu}=-\frac{i \nu}{2}(\hat{q}-\hat{Q})^{2}+h(\hat{Q})
$$

where the function $h$ is the (lower) symbol of the original Hamiltonian $\hat{H}$ in the canonical coherent state representation. Observe that $h(\hat{Q})$ is uniquely defined, no operator ordering ambiguities occur since the operators $\hat{Q}^{k}$ are commutative. In the operator formalism developed, the Wiener measure regularization is based on the important relation

$$
\lim _{\nu \rightarrow \infty}\left(q\left|e^{-i t \hat{H}_{\nu}}\right| q^{\prime}\right) \equiv \lim _{\nu \rightarrow \infty} K_{t}^{\nu}\left(q, q^{\prime}\right)=\left\langle q\left|e^{-i t \hat{H}}\right| q^{\prime}\right\rangle, \quad t>0
$$

Thus, the ill-defined formal path integral representation of the r.-h.s. is replaced by the convergent sequence of well-defined stochastic integrals in the l.-h.s. which, as has been argued above, are explicitely covariant under general coordinate transformations $q^{k} \rightarrow$ $\bar{q}^{k}(q)$. Note also that $\hat{H}_{\nu}^{\dagger} \neq \hat{H}_{\nu}$, and the immaginary part of the extended Hamiltonian $\hat{H}_{\nu}$ gives rise to the Wiener measure in the path integral representation. 
Now let $q^{i}$ be local coordinates on a symplectic manifold, with

$$
\left\{q^{j}, q^{k}\right\}_{q}=\omega^{j k}(q)
$$

The symplectic two-form $\omega=\omega_{i j} d q^{i} \wedge d q^{j}, \omega_{i k} \omega^{k j}=\delta_{i}^{j}$, is closed $d \omega=0$ (the Jacobi identity for the Poisson bracket (6)). A formal path integral quantization (11) (where the symplectic volume element and the symplectic one-form are modified according to the symplectic structure (6)) runs into notorious difficulties if one tries to relate it to the operator formalism. In particular, by means of the formal change of the integration variables to Darboux variables, the overlap function $\left\langle q \mid q^{\prime}\right\rangle$ (the integral kernel of the unit operator, $h=0$ ) seems always possible to be expressed as the integral (1) with the canonical symplectic structure. This is certainly not true. Coherent states on general manifolds do not coincide with the canonical coherent states. Therefore, the path integral measure must be regularized relative to general coordinate transformations on the phase space. A possible way to achieve this is to replace the formal, ill-defined Liouville measure by the Wiener measure with an appropriate metric. However, there is a great deal of freedom in choosing such a metric [2]. The overlap function $\left\langle q \mid q^{\prime}\right\rangle$ and, hence, the entire Hilbert space will depend on this choice.

The main goal of our work is to give a procedure of how the metric can be constructed and to show that the corresponding path integral is coordinate-free and determines a consistent quantum mechanics.

2. To avoid the difficult problem of quantizing general symplectic structure (6), we shall use the conversion formalism [4, 5]. The idea is to embed the symplectic manifold into a larger Euclidean (flat) phase space and impose special second-class constraints such that, when reduced on the constraint surface, the canonical symplectic structure of the embedding space turns into the original symplectic structure. The second-class constraint system is then replaced by an effective Abelian gauge theory, and the latter is quantized by the standard methods. The algebra of gauge invariant operators is then taken as a quantum algebra of observables associated with the symplectic structure (6). The representation (Hilbert) space of this algebra is a subspace of gauge invariant functions in the effective quantum gauge theory.

Let us turn to the details. Consider an extended Euclidean phase space spanned by the Cartesian coordinates $q^{i}$ and momenta $p_{i}$, and equipped with a canonical symplectic structure which we choose as $\left\{q^{i}, q^{j}\right\}=\left\{p_{i}, p_{j}\right\}=0$ and $\left\{q^{i}, p_{j}\right\}=\delta_{j}^{i}$. The second-class constraints are

$$
\varphi_{j}=p_{j}+\alpha_{j}(q)=0
$$

where $\alpha_{i}(q)$ are coefficients of the symplectic one-form $\alpha=\alpha_{i} d q^{i}$ and $d \alpha=\omega$ being the symplectic two-form. It is not hard to verify that $\Delta_{i j}=\left\{\varphi_{i}, \varphi_{j}\right\}=\omega_{i j}(q)$. The symplectic structure on the constraint surface is induced by the Dirac bracket $\{\cdot, \cdot\}_{D}=$ $\{\cdot, \cdot\}-\left\{\cdot, \varphi_{i}\right\} \Delta^{i j}\left\{\varphi_{j}, \cdot\right\}$, where $\Delta^{i k} \Delta_{k j}=\delta_{j}^{i}$. Eliminating the variables $p_{i}$ by means of the constraints and assuming the variables $q^{i}$ to be the coordinates on the physical phase space, we find that the induced symplectic structure coincides with the original one: $\left.\left\{q^{j}, q^{k}\right\}_{D}\right|_{\varphi=0}=\omega^{j k}(q)$. Thus, a Hamiltonian dynamics on a generic symplectic 
manifold can be described as a second-class constraint system with the action $S_{\varphi}=$ $\int\left(p_{j} \dot{q}^{j}-h(q)-\lambda^{j} \varphi_{j}\right) d t$ on an extended phase space.

To quantize the theory, the canonical variables $p_{j}$ and $q^{j}$ are promoted into selfadjoint operators satisfying the canonical commutation relations $([]=,i\{\}$,$) . One next$ introduces a new set of canonical variables $\theta^{j}$ associated with the second-class constraints $\varphi_{j}$ also satisfying the Heisenberg commutation relations $\left[\hat{\theta}^{j}, \hat{\theta}^{k}\right]=i \stackrel{\circ}{\omega}{ }^{j k}$ and commuting with $\hat{q}^{j}$ and $\hat{p}^{k}$. A general operator $\hat{A}$ can be represented in the form

$$
\hat{A}=\sum_{n} A_{j_{1} \cdots j_{n}}^{(n)}(\hat{p}, \hat{q}) \operatorname{sym}\left(\hat{\theta}^{j_{1}} \cdots \hat{\theta}^{j_{n}}\right) \equiv \sum_{n} A_{j_{1} \cdots j_{n}}^{(n)}(\hat{p}, \hat{q}) \hat{e}_{(n)}^{j_{1} \cdots j_{n}}
$$

where the symbol sym means a symmetrized product of the operators in the brackets. The physical subspace in the extended Hilbert space is selected by the gauge invariance condition

$$
\hat{\sigma}_{j}\left|\psi_{p h}\right\rangle=0
$$

where the generators of gauge transformations $\hat{\sigma}_{j}$ and the Hamiltonian in the extended Hilbert space are constructed to satisfy the conversion equations [4 5

$$
\left[\hat{\sigma}_{j}, \hat{\sigma}_{k}\right]=0, \quad\left[\hat{H}_{g}, \hat{\sigma}_{j}\right]=0
$$

subject to the boundary conditions $\hat{\sigma}_{j}^{(0)}=\hat{\varphi}_{j}, \hat{H}_{g}^{(0)}=H(\hat{q})$. In the classical limit, the dynamics of the physical degrees of freedom of the so constructed gauge theory coincides with that on the original symplectic manifold [5], that is, the correspondence principle is fulfilled.

To solve the conversion equations, one sets [6, 7] $\hat{\sigma}_{j}=\hat{\varphi}_{j}+A_{j}(\hat{q}, \hat{\theta}), A_{j}^{(0)}(\hat{q})=0$. The operators $\hat{q}^{j}$ commute, so we omit the hat while analyzing the equation for $\hat{A}_{j}=A_{j}(q, \hat{\theta})$ that results from $(\mathbb{1 0})$

$$
\partial_{j} \hat{A}_{k}-\partial_{k} \hat{A}_{j}-i\left[\hat{A}_{j}, \hat{A}_{k}\right]=\omega_{j k}(q) .
$$

Here $\partial_{j}=\partial / \partial q^{j}$. The solution is not unique. If $\hat{A}_{k}$ is a solution then for any $\hat{\Lambda}=\Lambda(q, \hat{\theta})$, the operator $\hat{A}_{k}^{\Lambda}=e^{i \hat{\Lambda}} \hat{A}_{k} e^{-i \hat{\Lambda}}-i e^{i \hat{\Lambda}} \partial_{k} e^{-i \hat{\Lambda}}$ is also a solution, provided this transformation does not violate the zero boundary condition $\hat{A}_{k}^{(0)}=0$. In fact, this condition imposes some restrictions on $\hat{\Lambda}$ discussed later.

An important point to observe is that the physical amplitudes do not depend on $\hat{\Lambda}$. Indeed, any physical amplitude reads $\left\langle\psi_{1}|\hat{O}| \psi_{2}\right\rangle$ where $\left|\psi_{1,2}\right\rangle$ are gauge invariant (physical) states determined by (9) and $\hat{O}$ is any operator satisfying the condition $\left[\hat{O}, \hat{\sigma}_{i}\right] \sim \hat{\sigma}_{i}$. If $\hat{A}_{k}$ is replaced by $\hat{A}_{k}^{\Lambda}$, then the new Abelian constraints are $\hat{\sigma}_{k}^{\Lambda}=e^{i \hat{\Lambda}} \hat{\sigma}_{k} e^{-i \hat{\Lambda}}$. Thus, the new sets of physical operators and states are obtained from the former ones by the unitary transformation $e^{i \hat{\Lambda}}$, that is, the physical amplitudes are not changed. It is therefore sufficient to solve the conversion equations modulo the $\Lambda$-transformations.

3. The right-hand side of Eq. (11) can be regarded as the field strength in the YangMills theory with an infinite dimensional gauge group generated by the operators $\hat{e}_{(n)}$. Introducing the Yang-Mills potential $\hat{C}_{k}=\alpha_{k}+\hat{A}_{k}$, we observe that the corresponding field strength vanishes if $\hat{A}_{k}$ satisfies (11). This means that the vector potential $\hat{C}_{k}$ must 
be a pure gauge, $\hat{C}_{k}=-i e^{i \hat{\Omega}} \partial_{k} e^{-i \hat{\Omega}}$, where $\hat{\Omega}=\Omega(q, \hat{\theta})$. Yet, this solution is determined modulo the gauge $(\Lambda$-)transformations. The operator $\hat{\Omega}$ satisfies only the condition that $\hat{C}_{k}^{(0)}=\alpha_{k}$ and this condition should not be violated under the gauge transformations of $\hat{C}_{k}$. For this reason, the pure gauge potential $\hat{C}_{k}$ can not be gauged to a vanishing potential (which does not satisfy the condition $\hat{C}_{k}^{(0)}=\alpha_{k}$ ).

The operators $\hat{e}_{(0)}$ and $\hat{e}_{(1)}$ are generators of the Heisenberg algebra which is a finite dimensional subalgebra of the algebra generated by all the $\hat{e}_{(n)}$. So we can always represent the gauge group element $e^{i \hat{\Lambda}}$ as the product $e^{i \hat{\Lambda}_{H}} e^{i \hat{\Lambda}_{\infty}}$, where $\hat{\Lambda}_{H}$ is an element of the Heisenberg algebra, and $\hat{\Lambda}_{\infty}=\sum_{n>1} \lambda^{(n)} \hat{e}_{(n)}$ (here we suppress the indices $j_{1}, \ldots, j_{n}$ ). The gauge transformation of $\hat{C}_{k}$ with the gauge group element $e^{i \hat{\Lambda}_{\infty}}$ leaves the component $\hat{C}_{k}^{(0)}$ untouched so it does not violate the required condition $\hat{C}_{k}^{(0)}=\alpha_{k}$. Thus, modulo gauge transformations, we may write $\hat{C}_{k}=-i e^{i \hat{\Omega}_{H}} \partial_{k} e^{-i \hat{\Omega}_{H}}$, where $\hat{\Omega}_{H}$ is an element from the Heisenberg algebra, or, equivalently,

$$
\hat{C}_{k}=\alpha_{k}+\hat{A}_{k}=\alpha_{k}(q)+a_{k j}(q) \hat{\theta}^{j}, \quad a_{k j} \stackrel{\circ}{\omega}^{j i} a_{i n}=\omega_{k n}, \quad \partial_{j} a_{k i}=\partial_{k} a_{j i} .
$$

The two latter relations follow from Eq.(11). If $Q^{j}=Q^{j}(q)$ are Darboux coordinates for the symplectic structure in question, then in local coordinates $a_{j k}=\partial_{j} Q^{n} \stackrel{\circ}{\omega}_{n k}$.

The gauge arbitrariness of the solution (12) is not yet exhausted. The gauge transformations from the Heisenberg group $\left(\hat{\Lambda}=\hat{\Lambda}_{H}\right)$ still apply. Representing the gauge group element as the product of operators $e^{i \lambda_{j}^{(1)} \hat{\theta^{j}}}$ (no summation over $j$ ) and the phase factor $e^{i \lambda^{(0)}}$ (associated with the unit operator in the Heisenberg algebra), we calculate the gauge transformation of the solution (12) explicitly. Assuming some set of the Darboux coordinates to represent the functions $a_{j k}$, we get that $Q^{j} \rightarrow Q_{\lambda}^{j}=Q^{j}+\stackrel{\circ}{\omega}^{j k} \lambda_{k}^{(1)}$ under the gauge transformation. The condition that $\hat{C}_{k}^{(0)}=\alpha_{k}$ should not be violated under any gauge transformation would yield that $\partial_{k} \lambda^{(0)}=f_{k}\left(\lambda^{(1)}, Q\right)$ (we omit the details) which specifies $\lambda^{(0)}$. The integrability condition for this equation leads to

$$
\partial_{i} Q_{\lambda}^{k} \stackrel{\circ}{\omega}_{k l} \partial_{j} Q_{\lambda}^{l}=\omega_{i j}
$$

Thus, $Q_{\lambda}^{j}$ is just another set of Darboux variables. This, of course, might be expected at the very beginning since the residual gauge transformations do not change the structure of the solution (12).

The gauge freedom in choosing the Darboux variables appears desirable. Whatever set of Darboux variables we use in practical calculations of the physical amplitudes, the result will not depend on it. Changing from one set of Darboux coordinates to another implies a corresponding modification of the gauge invariance condition. The physical states and operators are modified so that the physical amplitudes remain unchanged. This freedom also allows one to make the quantum theory globally well-defined [7].

4. The invariance of the theory under canonical transformations of the Darboux variables is crucial for the path integral formalism because one has to assume a specific form of the constraints in order to calculate the path integral. So we pick out some set 
of Darboux coordinates $Q^{j}$. The Hamiltonian of the effective gauge theory satisfying the conversion equation (10) has the form

$$
\hat{H}_{g}=H(\hat{\vartheta}), \quad \hat{\vartheta}^{i}=\operatorname{sym} q^{i}(\hat{\theta}+Q(q)),
$$

where the functions $q^{i}=q^{i}(Q)$ are inverse to $Q^{i}(q)$, i.e., $q^{i}(Q(q)) \equiv q^{i}$. We also assume some appropriate ordering of the operators $\hat{\vartheta}^{i}$ to make the Hamiltonian hermitian. Making use of the explicit form of the constraints, it is easy to prove that $\left[\hat{\sigma}_{j}, \hat{\vartheta}^{k}\right]=0$. The required boundary condition follows from the relation $\hat{\vartheta}^{(0) i}=q^{i}$.

The commutation relations of all canonical variables in the effective Abelian gauge theory are the standard ones, so we can construct the Wiener measure regularized path integral for the transition amplitude on the extended phase space along the lines discussed in Section 1. To simplify notation, we denote by $b^{\alpha}$ the set $q^{i}, p_{i}$ and $\theta^{i}$, and will write $\left\{b^{\alpha}, b^{\beta}\right\}=\stackrel{\circ}{\omega}{ }^{\alpha \beta}$. The amplitude $K_{t}^{\nu}\left(b, b^{\prime}\right)$ satisfies Eq.(3), where $q^{i}$ are now replaced by $b^{\alpha}$ and $h(b)$ is the symbol of the operator (14) in the canonical coherent state representation. The integral kernel $K_{t}^{\nu}\left(b, b^{\prime}\right)$ is not physical because it is not gauge invariant. To obtain the physical evolution operator, we make use of the projection method [8, 9]. Consider a unitary operator $\hat{U}(\xi)=e^{i \xi^{j} \hat{\sigma}_{j}}$ with $\xi^{j}$ being parameters. For any physical state we have $\hat{U}(\xi)|\psi\rangle=|\psi\rangle$. We define a projection operator $\hat{\mathcal{P}}=\int d \kappa(\xi) \hat{U}(\xi)$ where the measure $d \kappa(\xi)$ is normalized to unity, $\int d \kappa(\xi)=1$. Then $\hat{\mathcal{P}}^{\dagger}=\hat{\mathcal{P}}$ and $\hat{\mathcal{P}}^{2}=\hat{\mathcal{P}}$. In general, the zero eigenvalue of the constraint operators may lie in the continuum spectrum of some or all the constraints. In this case we take a sequence of the rescaled projection operators $c_{\delta} \hat{\mathcal{P}}_{\delta}, c_{\delta}>0$, as $\delta \rightarrow 0$, where $\hat{\mathcal{P}}_{\delta}=\int d \kappa_{\delta}(\xi) \hat{U}(\xi)$, and the measure is chosen so that $\hat{\mathcal{P}}_{\delta}$ projects the Hilbert space into a subspace where $\sum \hat{\sigma}_{i}^{2} \leq \delta^{2}$ for $0<\delta \ll 1$ [9].

So, our task is to develop a coordinate-free path integral representation of the gauge invariant transition amplitude $\left\langle q\left|\hat{\mathcal{P}} \hat{K}_{t} \hat{\mathcal{P}}\right| q^{\prime}\right\rangle=\left\langle q\left|\hat{K}_{t} \hat{\mathcal{P}}\right| q^{\prime}\right\rangle$. By construction, $\left[\hat{H}, \hat{\sigma}_{k}\right]=0$. To this end we consider the Schrödinger-like equation that depend on a set of general functions $\xi^{k}(t)$

$$
\begin{aligned}
i \partial_{t} \hat{K}_{t}^{\nu}[\xi] & =\left\{-\frac{i \nu}{2}(\hat{b}-\hat{B})^{2}+h(\hat{B})+\xi^{k}(t) \sigma_{k}(\hat{B})\right\} \hat{K}_{t}^{\nu}[\xi] \\
& \equiv\left\{\hat{H}_{\nu}+\xi^{k}(t) \sigma_{k}(\hat{B})\right\} \hat{K}_{t}^{\nu}[\xi]
\end{aligned}
$$

As before, we have extended the original Hilbert space to an irreducible representation space of the canonical operators $\left[\hat{B}^{\alpha}, \hat{P}_{\beta}\right]=i \delta_{\beta}^{\alpha},\left[\hat{B}^{\alpha}, \hat{B}^{\beta}\right]=\left[\hat{P}_{\alpha}, \hat{P}_{\beta}\right]=0$, while the original canonical operators now have a reducible representation $\hat{b}^{\alpha}=-\stackrel{\circ}{\omega}{ }^{\alpha \beta} \hat{P}_{\beta}+\frac{1}{2} \hat{B}^{\alpha}$. The functions $h$ and $\sigma$ are the (lower) symbols of the Hamiltonian $\hat{H}$ and constraints $\hat{\sigma}_{k}$ in the original Hilbert space $\mathcal{H}_{\infty}$. With this choice we can prove the following important property of the solution to Eq. (15) with the intitial condition $\hat{K}_{t=0}^{\nu}[\xi]=\hat{\mathbb{I}}_{\nu}$

$$
\left(b\left|\hat{K}_{t}^{\nu}[\xi]\right| b^{\prime}\right)=\left(b\left|T e^{-i \int_{0}^{t} d \tau\left[\hat{H}_{\nu}+\xi^{k}(\tau) \sigma_{k}(\hat{B})\right]}\right| b^{\prime}\right) \rightarrow\left\langle b\left|T e^{-i \int_{0}^{t} d \tau\left[\hat{H}+\xi^{k}(\tau) \hat{\sigma}_{k}\right]}\right| b^{\prime}\right\rangle
$$

as $\nu \rightarrow \infty$, for all $t>0$. Here $\left.\hat{B}^{k} \mid b\right)=b^{k} \mid b$ ). Averaging Eq.(16) over the functions $\xi^{k}$ we obtain the sought-for representation of the physical transition amplitude

$$
\left\langle b\left|\hat{K}_{t} \hat{\mathcal{P}}\right| b^{\prime}\right\rangle=\lim _{\nu \rightarrow \infty} \int d \rho(\xi)\left(b\left|\hat{K}_{t}^{\nu}[\xi]\right| b^{\prime}\right),
$$


where $d \rho(\xi)$ stands for a normalized measure for functions $\xi^{k}(\tau)$ on the interval $\tau \in$ $[0, t]$. In particular we may take the white-noise measure $d \rho(\xi)=d \rho_{\nu}(\xi) \equiv \mathcal{D} \xi e^{-\frac{1}{2 \nu} \int \xi^{2} d \tau}$, $\nu \rightarrow \infty$, or a non-pinned Wiener measure [11. As a point of fact, the only condition the measure should satisfy is that it must provide at least one action of the projection operator $\mathcal{P}$ within the time interval $[0, t]$. By going over to the path integral representation in the l.-h.s. of Eq. (17), we derive our final result

$$
K_{t}\left(b, b^{\prime}\right)=\lim _{\nu \rightarrow \infty} N_{\nu}(t) \int d \rho(\xi) \int_{b(0)=b^{\prime}}^{b(t)=b} d \mu_{W}^{\nu}(b) e^{i \int_{0}^{t}\left(\frac{1}{2} b^{\alpha} \stackrel{o}{\omega}_{\alpha \beta} d b^{\beta}-\left[h(b)+\xi^{k} \sigma_{k}(b)\right] d \tau\right)} .
$$

The path integral (18) with the measure $d \rho(\xi)$ included has a genuine (finite and countably additive) measure which is covariant under general coordinate transformations. It provides the sought for path integral formalism for general Hamiltonian systems, which was our main goal.

To relate the path integral (18) to the original phase space manifold, one can make a change of variables (which, in this case, is a well-defined procedure!) $(q, p, \theta) \rightarrow(q, \sigma, \vartheta)$ where $\vartheta^{k}=q^{k}(\theta+Q(q))$ or, equivalently, $\theta^{k}=\int_{q}^{\vartheta} d \bar{q}^{n} a_{n l} \stackrel{\stackrel{\circ}{\omega}}{l k}=\int_{q}^{\vartheta} d Q^{k}$ for any integration contour. In this representation, the canonical pairs $(q, \sigma)$ are nonphysical degrees of freedom due to the translational gauge symmetry $q \rightarrow q+\delta \xi$. The variables $\vartheta$ are gauge invariant and represent the physical degrees of freedom, and their Poisson bracket gives the original symplectic structure $\left\{\vartheta^{i}, \vartheta^{j}\right\}=\omega^{i j}(\vartheta)$ and $h=h(\vartheta)$ by construction. If the integral over $q, \sigma$ and $\xi$ is done, the result will be given by the exponential of the classical action averaged with the induced non-canonical, non-flat Wiener measure on the original symplectic manifold. The induced measure will specify the unit operator kernel $\left\langle\vartheta \mid \vartheta^{\prime}\right\rangle$ and generalized coherent-state representation of the non-canonical symplectic structure. This technical problem will be studied elsewhere.

5. In conclusion, we would like to emphasize the following. A representation of the quantum mechanical evolution as a sum over paths should not depend on coordinates because the geometrical notion of a path on the symplectic manifold is coordinate independent (a path is specified by its tangent vector). Thus, in order to determine a formulation of quantum mechanics via the path integral on the symplectic manifold, or, in other words, to quantize a general Hamiltonian system on the manifold by the path integral method, it is fundamental that the corresponding functional measure is coordinate-free. The latter, in our opinion, requires an (induced) metric on the symplectic manifold. We have given a constructive way of how this regularization can be obtained. The procedure developed here provides a solution to the long-standing problem of constructing a rigorous coordinate-free path integral formalism on symplectic manifolds. The above idea can also be successfully applied to the path integral quantization of gauge and constrained systems, in general (in a flat phase space) [10, 11]. The operator technique established in Section 4 can be used to greatly simplify the Wiener measure regularization of the path integral in gauge theories and second-class constrained systems proposed in our earlier works [10, 11]. It is also allows one to generalize the result of [10] to most general (open) first-class constraint algebras. 
Thus, the coordinate-free path integral formalism based on the Wiener measure regularization can be regarded as a universal, self-consistent, path-integral quantization technique.

\section{Acknowledgment}

I.A. Batalin is thanked for useful comments and references on the conversion method. S.V.S. is grateful to L. Baulieu and H. Kleinert for kind hospitality at the University Paris VI and the Free University of Berlin, and to CNRS (France) and DFG (Germany) for financial support.

\section{References}

[1] P.A.M. Dirac, The Principles of Quantum Mechanics, (Oxford University Press, Oxford, Fourth Edition, 1976), p. 114.

[2] J.R. Klauder, Ann. Phys. (N.Y.) 188 (1988) 128.

[3] J. Gervais and A. Jevicki, Nucl.Phys. B110 (1976) 93;

L.V. Prokhorov, Sov. J. Part. Nucl. Phys. 13(5) (1982) 456.

[4] L.D. Faddeev and S. Shatashvili, Phys.Lett. B167 (1986) 225.

[5] I.A. Batalin and E.S. Fradkin, Nucl.Phys. B326 (1989) 701; B279 (1987) 514.

[6] I.A. Batalin and I.V. Tyutin, Int.J.Mod.Phys. A6 (1991) 3255.

[7] E.S. Fradkin and V.Ya. Linetsky, Nucl.Phys. B431, 569 (1994).

[8] L.V. Prokhorov and S.V. Shabanov, Sov.Phys.Uspekhi 34(2), 108 (1991).

[9] J.R. Klauder, Ann.Phys. 254, 419 (1997).

[10] J.R. Klauder and S.V. Shabanov, Phys.Lett. B398, 116 (1997).

[11] J.R. Klauder and S.V. Shabanov, Nucl.Phys. B511, 713 (1998). 